\title{
A PALEY-LIKE GRAPH IN CHARACTERISTIC TWO
}

\author{
ANDREW THOMASON
}

\begin{abstract}
The Paley graph is a well-known self-complementary pseudorandom graph, defined over a finite field of odd order. We describe an attempt at an analogous construction using fields of even order. Some properties of the graph are noted, such as the existence of a Hamiltonian decomposition.
\end{abstract}

\section{INTRODUCTION}

The well-known Paley graph is a pseudo-random graph whose vertex set is the finite field $F_{q}=G F(q)$ of order $q \equiv 1(\bmod 4)$. The pair of vertices $a, b$ is joined by an edge if $a-b$ is a square in $F_{q}$. Since -1 is a square the graph is well defined. It follows from elementary properties of the quadratic character that the Paley graph is vertex-transitive, self-complementary, and each edge is in $(q-5) / 4$ triangles. A graph of order $q$ in which every edge is in $(q-5) / 4$ triangles and whose complement has the same property is sometimes called a conference graph. The Paley graph is thus ipso facto a pseudo-random graph, as explained in detail in [12], and in a somewhat less quantitative fashion in Chung, Graham and Wilson in [3].

The other odd prime powers, namely those where $q \equiv 3(\bmod 4)$, cannot be used to construct Paley graphs since -1 is not a square. However this very property allows the construction of a tournament, or oriented complete graph, on the vertex $F_{q}$ by inserting an edge oriented from $a$ to $b$ if $a-b$ is a square. Since -1 is not a square, exactly one of $a-b$ and $b-a$ is a square, so we do indeed construct a tournament (Graham and Spencer [6]).

The property of pseudo-randomness, even when quantified, does not suffice to give all the information that one would like to have about the Paley graphs; in particular, it is not known what the clique number is. When $q$ is prime the calculation reduces to difficult and so far unsolved problems involving the estimation of character sums (though if $q$ is a square the clique size is exactly $\sqrt{q}$ ). For more information, see [13, Section 2.5.1].

As regards Hamiltonian cycles, the Paley graphs hold fewer secrets. If $q$ is a prime then the Paley graph is a circulant graph and, since the edges of a given distance in a circulant of prime order form a Hamiltonian cycle, it follows that the Paley graph in this case is not just Hamiltonian but it has a Hamiltonian decomposition, that is, its edge set is the union of edge-disjoint Hamiltonian cycles.

In a finite field of characteristic two, every element is a square, and the definition of the Paley graph is of little value. From the graph theoretical point of view, though, characteristic two has some innate attraction. In

Date: 6th March 2015.

2000 Mathematics Subject Classification. Primary 05C99; Secondary 05C25,05C45. 
this note we describe an attempt to find different graphs, similar in spirit to the Paley graphs but defined in relation to the field $F_{q}$ for even $q$, which are vertex-transitive and self-complementary. We might even hope to find a graph which is a conference graph, or which is more easily analysed than the Paley graph. Whilst these more ambitious aims are not realised, we do describe the more accessible properties of the graphs.

\section{Definition}

We begin with a definition of the graphs, and defer to $\$ 3$ a discussion of what lies behind it. Choose as vertex set $V$ the elements of $P G(1, q)$, the projective space of dimension one over $F_{q}$. We label the elements of $V$ in the natural way, namely

$$
V=\left\{\left(\begin{array}{l}
1 \\
0
\end{array}\right),\left(\begin{array}{l}
0 \\
1
\end{array}\right),\left(\begin{array}{l}
1 \\
1
\end{array}\right), \ldots,\left(\begin{array}{l}
x \\
1
\end{array}\right), \ldots\right\}=\{\infty, 0,1, \ldots, x, \ldots\} .
$$

Given an element $x \in F_{q}$ its trace is defined to be $\operatorname{tr}(x)=x+x^{2}+x^{4}+$ $\ldots+x^{q / 2}$. Let $q=2^{k}$ and let $a$ be an element of $F_{q}$ with $\operatorname{tr}(a)=1$. For even $k$ we define a graph $G_{k}(a)$ on the vertex set $V$ by

$$
x y \in E\left(G_{k}(a)\right) \quad \text { if } \quad \operatorname{tr}\left(\frac{x y+x+a}{x+y}\right)=0 .
$$

For odd $k$ we define a tournament $G_{k}(a)$, having an edge directed from $x$ to $y$ whenever the same equation is satisfied.

We shall show in 4.1 that $G_{k}(a)$ is well defined. Moreover, although $G_{k}(a)$ as a labelled graph depends on the value of $a$ (for example, the neighbourhood of the vertex 0 is the set of elements $y$ such that $\operatorname{tr}(a / y)=0)$, we shall show in $\$ 5$ that all the graphs (or tournaments) so defined are isomorphic. This allows us to refer to any member of this collection of graphs as the graph $G_{k}$ when there is no danger of confusion.

At first appearance the definition of the graph $G_{k}$ looks somewhat contrived. We attempt in 93 to show that the definition does in fact arise fairly naturally. Having made a few elementary remarks about the properties of $F_{q}$ (in 84 ) we establish in \$5 that $G_{k}(a)$ is a vertex-transitive self-complementary graph whose isomorphism class is independent of $a$, as claimed. Finally, we explore some of the further properties of the graph $G_{k}$; in particular we show that it is a pseudo-random graph, having a Hamiltonian decomposition.

\section{BACKGROUND}

The Paley graph is a circulant graph when $q$ is prime; that is, its vertices may be labelled $\{0,1, \ldots, q-1\}$, and whether $x y$ is an edge depends only on the difference $|x-y|$. It is therefore necessarily vertex-transitive, and it is also self-complementary. A regular self-complementary graph has order $\equiv 1(\bmod 4)$, and obviously there is no such graph with vertex set $F_{q}$ when $q$ is even. We set out to define a circulant graph on vertex set $P G(1, q)$.

The group $P S L(2, q)$, comprising the $2 \times 2$ matrices of determinant one, acts on $V=P G(1, q)$. As usual, we associate with the matrix $\left(\begin{array}{ll}a & b \\ c & d\end{array}\right)$ the Möbius, or linear fractional, map $z \mapsto(a z+b) /(c z+d)$. We use two simple facts about these maps; that they form a group, and (for this background discussion) that a map is determined by its action on any three points. For 
convenience and completeness we assume only a minimal familiarity with properties of finite fields. Much more can be found in the classical algebraic text of Dickson [5] or the more recent and geometrical Hirschfeld [7]. Both these authors pay attention to the characteristic two case needed here.

In order to begin constructing a circulant on $V$ we need a Möbius transformation of order $q+1$. It is not hard to show, though we don't need this fact, that every transformation with no fixed points is conjugate to one of the form $z \mapsto a /(z+1)$ such that the equation $x^{2}+x=a$ has no solution in $F_{q}$. Let us then consider such a map. The condition that $x^{2}+x=a$ has no solution is equivalent to the condition $\operatorname{tr}(a)=1$ (see 44.1 ). Amongst such transformations there exist some of order $q+1$ (see $\$ 4.2$ ).

Take such a transformation $\alpha$. Then $V=\left\{\infty, \alpha(\infty), \alpha^{2}(\infty), \ldots, \alpha^{q}(\infty)\right\}$. For convenience, we write $v_{i}=\alpha^{i}(\infty)$, so $V=\left\{v_{0}, v_{1}, \ldots, v_{q}\right\}$. Notice that, for example, $v_{1}=\alpha(\infty)=0$ and $v_{2}=\alpha(0)=a$. Moreover $\alpha^{-1}(z)=1+a / z$, so $v_{q}=\alpha^{q}(\infty)=\alpha^{-1}(\infty)=1$ and $v_{q-1}=\alpha^{-1}(1)=1+a$. It is easily verified, by induction on $i$, that $v_{q-i}=1+v_{i}$ (the induction step being $\left.v_{q-i-1}=\alpha^{-1}\left(v_{q-i}\right)=1+a / v_{q-i}=1+a /\left(1+v_{i}\right)=1+\alpha\left(v_{i}\right)=1+v_{i+1}\right)$. Subscripts may be reduced modulo $(q+1)$, so we write $v_{-i}=1+v_{i}$.

We may, therefore, define a circulant graph on $V$ as follows. Choose a map $f: F_{q} \rightarrow F_{2}$, to be specified later. The neighbours of $\infty=v_{0}$ will be those $v_{i}$ for which $f\left(v_{i}\right)=0$. In general, $v_{i} v_{j}$ will be an edge if $v_{0} v_{j-i}$ is an edge, which is to say, if $f\left(v_{j-i}\right)=0$. In order that the graph be well defined we must ensure that $f\left(v_{i-j}\right)=f\left(v_{j-i}\right)$, which we have seen is equivalent to $f(x+1)=f(x)$. (This section is just to motivate the earlier definition, so we ignore tournaments here.)

Let us see how to compute whether $x y$ is an edge, given $x, y \in V$. Let $x=v_{i}$ and $y=v_{j}$. Then $x y$ will be an edge if $f\left(v_{i-j}\right)=f\left(v_{j-i}\right)=0$. Now $v_{i-j}=\alpha^{-j}(x)$. We claim that the map $\alpha^{-j}$ is identical to the Möbius map $\beta(z)=(z y+z+a) /(z+y)$, and so $v_{i-j}=\beta(x)=(x y+x+a) /(x+y)$. To check the claim, it suffices to show that $\alpha^{-j}$ and $\beta$ act identically on the three distinct points $v_{j-1}, v_{j}$ and $v_{j+1}$. Now $\alpha^{-j}$ maps these points to $v_{-1}=1, v_{0}=\infty$ and $v_{1}=0$. But $v_{j}=y, v_{j-1}=\alpha^{-1}(y)=1+a / y$ and $v_{j+1}=\alpha(y)=a /(y+1)$. Thus $\beta\left(v_{j-1}\right)=\beta(1+a / y)=1, \beta\left(v_{j}\right)=\beta(y)=\infty$ and $\beta\left(v_{j+1}\right)=\beta(a /(y+1))=0$, proving the claim. We conclude that $x y$ is an edge if $f((x y+x+a) /(x+y))=f\left(v_{j-i}\right)=0$.

The map $v_{i} \mapsto v_{2 i}$ is a permutation of $V$ which leaves $v_{0}$ fixed. An easy way to ensure that our circulant graph is self-complementary is to arrange that this map interchanges the graph with its complement. So we wish to arrange that if $x=v_{i}$ then $f\left(v_{2 i}\right) \neq f(x)$, or, equivalently, $f\left(v_{2 i}\right)+f(x)=1$. If we put $j=-i$ then $v_{2 i}=v_{i-j}$, and using the calculation in the previous paragraph with $y=v_{-i}=1+x$, we see that $v_{2 j}=(x y+x+a) /(x+y)=$ $x^{2}+a$.

Therefore this procedure will yield a self-complementary vertex-transitive graph if we select a function $f: F_{q} \rightarrow F_{2}$ such that $f(x)=f(x+1)$ and $f(x)+f\left(x^{2}+a\right)=1$ for all $x \in F_{q}$. An obvious choice is $f=\operatorname{tr}$. In fact, this is the only natural choice which does not depend on $a$ itself; for we may assume that $f(0)=0$, and then we must have $f(a)=1$ for all $a$ to which the discussion applies, namely those $a$ for which $\operatorname{tr}(a)=1$ and $\alpha$ has order 
$q+1$. This is close to requiring $f(a)=1$ whenever $\operatorname{tr}(a)=1$, which in turn implies $f=\operatorname{tr}$ because $f$ must be zero on exactly half the elements of $F_{q}$.

So by the process described we arrive at the definition of the graph $G_{k}(a)$.

3.1. Other possibilities. Aiming for a circulant is not a priori the right thing to do; the Paley graphs are circulants if $q$ is prime but not in general. However, in order that the group $\operatorname{PSL}(2, q)$ have a nice action on our graph we should choose its edge set to be a union of orbits of elements of $\operatorname{PSL}(2, q)$. We also want the graph to be self-complementary and to have some Möbius map interchanging the graph and its complement. As we shall see below, a great number of Möbius maps have order $q+1$, and so any graph of this more general kind is likely to be circulant.

\section{FIELD WORK}

Here we make further, standard and elementary, calculations over finite fields to justify some earlier remarks. A full treatment of these matters can be found in Lidl and Niederreiter [9].

4.1. Trace comments. The trace map is defined by $\operatorname{tr}(a)=a+a^{2}+a^{4}+$ $\ldots+a^{q / 2}$. Thus $\operatorname{tr}(a)^{2}=\operatorname{tr}(a)$ so $\operatorname{tr}(a) \in F_{2}$. Moreover trace is a linear map. There is a distinction between even $k$ and odd $k$, because

$$
\operatorname{tr}(1)=1+1^{2}+1^{4}+\ldots+1^{k-1}= \begin{cases}0 & \text { if } k \text { is even } \\ 1 & \text { if } k \text { is odd }\end{cases}
$$

Since trace is a linear map,

$$
\operatorname{tr}\left(\frac{x y+x+a}{x+y}\right)+\operatorname{tr}\left(\frac{y x+y+a}{y+x}\right)=\operatorname{tr}(1) .
$$

It follows that the definition in $\$ 2$ determines a graph if $k$ is even and a tournament if $k$ is odd, as claimed.

The map $\operatorname{tr}: F_{q} \rightarrow F_{2}$ is surjective, since trace, being a polynomial of degree lower than $q$, cannot annihilate $F_{q}$. Let $T_{i}=\operatorname{tr}^{-1}(i), i=0,1$. Then $T_{0}$ is the kernel of trace; since the map is surjective, we have $\operatorname{dim} T_{0}=k-1$ and so $\left|T_{0}\right|=\left|T_{1}\right|=2^{k-1}=q / 2$.

Now $\operatorname{tr}\left(a^{2}\right)=\operatorname{tr}(a)$, or $\operatorname{tr}\left(a^{2}+a\right)=0$. The map $x \mapsto x^{2}+x$ is also a linear map $F_{q} \rightarrow F_{q}$. Its kernel is $F_{2}$ so its image has dimension $k-1$. But its image contains $T_{0}$. Therefore its image is $T_{0}$; in particular, for every element $c$ with $\operatorname{tr}(c)=0$ there exists an element $b \in F_{q}$ with $b^{2}+b=c$. There are two solutions to this quadratic equation, the other being $b+1$. Thus if $k$ is even and $\operatorname{tr}(1)=0$ the two solutions have the same trace, whereas if $k$ is odd the solutions have different traces.

4.2. Möbius comments. Our aim here is to identify a suitable element $a \in F_{q}$ with which to carry out the above construction. Note that, for any $a$ with $\operatorname{tr}(a)=1$, then the equation $z^{2}+z+a=0$ has no solution in $F_{q}$, because $\operatorname{tr}\left(z^{2}+z+a\right)=\operatorname{tr}(a)=1$. Therefore the equation has a root $\lambda$ in $F_{q^{2}}$. It follows that $\bar{\lambda}=\lambda^{q}$ is the other root, because $\bar{\lambda}^{2}+\bar{\lambda}+a=\left(\lambda^{2}+\lambda+a\right)^{q}$.

Let $k$ be the order of the element $\bar{\lambda} / \lambda$ in $F_{q^{2}}$. Then $1=(\bar{\lambda} / \lambda)^{k}=\lambda^{k(q-1)}$, but also $\lambda^{q^{2}-1}=1$, so $k \mid(q+1)$ (in particular, if $q+1$ is a Fermat prime then $\bar{\lambda} / \lambda$ has order $q+1)$. 
Now let $k>2$ be any factor of $q+1$. Let $g$ be a primitive root for $F_{q^{2}}$. Then the cyclic group $\left\langle g^{q-1}\right\rangle$ of order $q+1$ has exactly $\phi(k)$ elements of order $k$, where $\phi$ is Euler's function. Let $\mu=g^{t(q-1)}$ be an element of order $k$ in $\left\langle g^{q-1}\right\rangle$. Then $\mu^{q-1}=g^{t(q-1)^{2}}=g^{-2 t(q-1)}$. Therefore $\mu \notin F_{q}$, for otherwise $\mu^{q-1}=1$ which would imply $(q+1) \mid 2 t$, which in turn would imply that $\mu^{2}=1$, contradicting $k>2$.

Given $\mu=g^{t(q-1)}$ as described, let $\nu=g^{t}$. Let $b=\nu+\bar{\nu}$, where $\bar{\nu}=\nu^{q}$. Then $\bar{b}=b^{q}=\bar{\nu}+\nu=b$, so $b \in F_{q}$. Put $\lambda=\nu / b$. Thus $\lambda+\bar{\lambda}=1$, and the element $\bar{\lambda} / \lambda=\bar{\nu} / \nu=\mu$ has order $k$. Let $\lambda \bar{\lambda}=a$; since $a^{q}=a$ we have $a \in F_{q}$. Moreover $\lambda^{2}+\lambda+a=0$, so $\operatorname{tr}(a)=\lambda+\lambda^{q}=1$.

We summarize as follows. Every element $a$ of trace 1 in $F_{q}$ satisfies an equation $\lambda^{2}+\lambda+a=0$ where $\lambda \in F_{q^{2}}$ and the order of $\bar{\lambda} / \lambda$ divides $q+1$. Conversely, for every factor $k>2$ of $q+1$ there exists such an $a$ such that $\bar{\lambda} / \lambda$ has order $k$.

In particular, there exists an $a$ such that $\bar{\lambda} / \lambda$ has order $q+1$. For such an $a$, consider the map $\alpha: z \rightarrow a /(z+1)$ and its associated matrix $\left(\begin{array}{ll}0 & a \\ 1 & 1\end{array}\right)$. This matrix has eigenvectors $\left(\begin{array}{l}\lambda \\ 1\end{array}\right)$ and $\left(\begin{array}{l}\bar{\lambda} \\ 1\end{array}\right)$ with eigenvalues $\bar{\lambda}$ and $\lambda$ respectively. Now $\infty=\left(\begin{array}{l}1 \\ 0\end{array}\right)=\left(\begin{array}{l}\lambda \\ 1\end{array}\right)+\left(\begin{array}{c}\bar{\lambda} \\ 1\end{array}\right)$. Therefore the result of applying the map $z \mapsto a /(z+1)$ to $\infty k$ times is $\bar{\lambda}^{k}\left(\begin{array}{l}\lambda \\ 1\end{array}\right)+\lambda^{k}\left(\begin{array}{c}\bar{\lambda} \\ 1\end{array}\right)$. This equals $\left(\begin{array}{l}1 \\ 0\end{array}\right)$ only if $\bar{\lambda}^{k}+\lambda^{k}=0$, which is to say $(\bar{\lambda} / \lambda)^{k}=1$. Since $\bar{\lambda} / \lambda$ has order $q+1$, the vertex $\infty$ is in an $\alpha$-orbit of size $q+1$. Thus there do exist elements $a$ for which the graph $G_{k}(a)$ is a self-complementary circulant graph, as described in 93 .

\section{Elementary properties}

Some of the more accessible properties of $G_{k}$ can now be described.

5.1. Isomorphisms. Let $b \in F_{q}$. The map $x \mapsto x+b$ is a permutation of $F_{q}$. Moreover

$$
\operatorname{tr}\left(\frac{(x+b)(y+b)+(x+b)+a}{(x+b)+(y+b)}\right)=\operatorname{tr}\left(\frac{x y+x+b^{2}+b+a}{x+y}\right)+\operatorname{tr}(b) .
$$

Suppose that $k$ is odd, that is, $\operatorname{tr}(1)=1$. Let $a$ and $a^{\prime}$ be two elements of $T_{1}$. Then $\operatorname{tr}\left(a+a^{\prime}\right)=0$, and by the remarks in 4.1. there exists an element $b$ with $b^{2}+b+a=a^{\prime}$ and $\operatorname{tr}(b)=0$. Therefore ( $\dagger$ ) shows that the map $x \mapsto x+b$ is an isomorphism $G_{k}\left(a^{\prime}\right) \rightarrow G_{k}(a)$. Moreover, since $1^{2}+1+a=a$, by $(\dagger)$ the map $x \mapsto x+1$ is an orientation-reversing bijection of the vertex set, because $\operatorname{tr}(1)=1$. It follows that the tournaments defined in $\oint_{2}$ are isomorphic to each other and are self-complementary.

Now let $k$ be even. We showed in $\$ 4$ that there is some element $a$ for which the map $\alpha: z \rightarrow a /(z+1)$ has order $q+1$ and $\operatorname{tr}(a)=1$. Let $a^{\prime}$ be any other element of $T_{1}$. Let $c=a^{\prime}-a$. Again, by the remarks in 4.1, there exists an element $b$ with $b^{2}+b+a=a^{\prime}$. Now either $\operatorname{tr}(b)=0$, in which case $(\dagger)$ shows that the map $x \mapsto x+b$ is an isomorphism $G_{k}\left(a^{\prime}\right) \rightarrow G_{k}(a)$, or $\operatorname{tr}(b)=1$, in which case the map $x \mapsto x+b$ is an isomorphism between $G_{k}\left(a^{\prime}\right)$ and the complement of $G_{k}(a)$. But $G_{k}(a)$ is vertex-transitive and self-complementary, 
as shown in $\oint_{3}$. Therefore the graphs defined in 92 are isomorphic to each other, being both vertex-transitive and self-complementary.

5.2. Automorphisms. Let $a \in F_{q}$ have trace one. The Möbius map $z \mapsto$ $a /(z+1)$ is a permutation of $V$. It is also an automorphism of the graph $G_{k}(a)$, because

$$
\frac{\frac{a}{x+1} \cdot \frac{a}{y+1}+\frac{a}{x+1}+a}{\frac{a}{x+1}+\frac{a}{y+1}}=\frac{x y+x+a}{x+y} .
$$

This, of course, is just the automorphism $\alpha$ that was built into the definition of $G_{k}(a)$.

In the graph case, the map $z \mapsto z+1$ is also an automorphism, being the map $v_{i} \mapsto v_{-i}$.

5.3. Co-degrees. The co-degree of a pair $x, y$ of vertices is the number of their common neighbours. As mentioned earlier, a $q / 2$-regular graph of order $q+1$ is a conference graph if every pair $x, y$ has codegree $q / 4-\epsilon$, where $\epsilon=0$ or 1 according as $x$ and $y$ are not adjacent or are adjacent.

The present graphs do not quite satisfy this condition but come close. Let us compute the co-degree of $x, y$ in $G_{a}(q)$. By the rotational symmetry we may assume that $y=\infty$. A vertex $w \notin\{\infty, x\}$ is joined to $\infty$ if $\operatorname{tr}(w)=0$ and to $x$ if $\operatorname{tr}((x w+x+a) /(x+w))=0$. Let $\psi: F_{q} \rightarrow\{-1,1\}$ be the additive character $\psi(z)=(-1)^{\operatorname{tr}(z)}$. If $\ell$ is the co-degree of $x, y$, then there are $q / 2-\epsilon-\ell$ vertices joined to $x$ but not to $y$, with the same number joined to $y$ but not $x$. So we have

$$
\sum_{w \in F_{q}, w \neq x} \psi(w) \psi\left(\frac{x w+x+a}{x+w}\right)=q-1-4(q / 2-\epsilon-\ell) .
$$

Thus, writing $K$ for the sum on the left, we have $\ell=q / 4-\epsilon+(K+1) / 4$.

Using the substitutions $w=z+x$ and $b=x^{2}+x+a$ we have

$$
K=\sum_{z \in F_{q}-\{0\}} \psi(z+x) \psi\left(x+\frac{b}{z}\right)=\sum_{z \in F_{q}-\{0\}} \psi\left(z+\frac{b}{z}\right) .
$$

Therefore $K$ is a Kloosterman sum; see Lidl and Niederreiter [9, Section 5.5] for a discussion. In particular, $|K| \leq 2 \sqrt{q}$ ([9, Theorem 5.45]). This was proved by Carlitz and Uchiyama [4], extending the proof by Weil [14] to even q. A self-contained proof, based on Stepanov [11], appears in Schmidt [10, Chapter 2].

In the case that $G_{k}$ is a graph we have $q=2^{k}$ where $k$ is even, and so $\sqrt{q}$ is an even integer. Therefore every co-degree is at most $q / 4+\sqrt{q} / 2$.

5.4. Pseudo-randomness. For our purposes, the import of the preceeding estimate of co-degrees is that the graph $G_{k}$ is pseudo-random. Specifically, it is $\left(1 / 2, q^{3 / 4}\right)$-jumbled, meaning that, for every induced subgraph $H \subset$ $G_{k},\left|e(H)-\frac{1}{2}\left(\begin{array}{c}|H| \\ 2\end{array}\right)\right| \leq q^{3 / 4}|H|$ holds. This follows comfortably from [12, Theorem 1.1] using the bound $q / 4+\sqrt{q} / 2$ for co-degrees.

From this it follows that $G_{k}$ enjoys all the usual consequences of pseudorandomness, such as expansion, having about the expected number of induced subgraphs of any given kind, and so on. 
Another approach to showing that $G_{k}$ is pseudo-random would be to estimate the eigenvalues, which are of course available in a reasonably explicit form given that $G_{k}$ is a circulant. However the present approach via codegrees is quick and effective.

5.5. Hamiltonian decompositions. As mentioned above, the Paley graph of order $q$ has a Hamiltonian decomposition when $q$ is prime because it is a circulant of prime order, and likewise so is $G_{k}$ if $q+1$ is a Fermat prime, though there seems to be a limited supply of these.

What about non-prime orders? For the Paley graph, there is always a Hamiltonian decomposition, as shown by Alspach, Bryant and Dyer [1]. The graphs $G_{k}$ too have a Hamiltonian decomposition, at least if $k$ is large. This follows from the deep work of Kühn and Osthus [8]. Theorem 1.2 of [8] states that there is some number $\tau>0$ such that, provided $G_{k}$ is a robust $(\tau / 3, \tau)$-expander, then $G_{k}$ has a Hamiltonian decomposition for large $k$. This condition requires that, for every subset $S$ of the vertices of $G_{k}$ with $\tau q \leq|S| \leq(1-\tau) q$, there are at least $|S|+\tau q / 3$ vertices of $G_{k}$ having at least $\tau q / 3$ neighbours in $S$. The condition is comfortably satisfied by $G_{k}$ because it is $\left(1 / 2, q^{3 / 4}\right)$-jumbled (using simple standard properties of such graphs [12]). The decomposition is, of course, not explicit but there is a polynomial time algorithm for finding it.

\section{AcKnowledgements}

Thanks are due to Robin Chapman for his comments. In particular he suggests another description of the graph $G_{k}$, from a field theoretic, rather than a geometric, viewpoint. The line $P G(1, q)$ can be identified in a natural way with the quotient group $F_{q^{2}}^{*} / F_{q}^{*}$, so we can consider graphs with this as its vertex set. Let $\lambda \in F_{q^{2}}^{*}$. Given $u, v \in F_{q^{2}}^{*}$ then the equivalence classes $[u],[v]$ are vertices of a graph $H_{\lambda}$, and we join $[u]$ to $[v]$ if $\operatorname{tr}\left(T\left(\lambda u^{q} v\right) / T(\lambda) T\left(u^{q} v\right)\right)=0$, where $T(x)=x+x^{q}$ is the trace map $F_{q^{2}}^{*} \rightarrow F_{q}^{*}$. Chapman [2] shows that $H_{\lambda}$ is well defined and isomorphic to $G_{k}$.

\section{REFERENCES}

[1] B. Alspach, D. Bryant and D. Dyer, Paley graphs have Hamilton decompositions, Discrete Math. 312 (2012), 113-118.

[2] R.J. Chapman, Thomason's Paley-type graph, (manuscript).

[3] F.R.K. Chung, R.L. Graham and R.M. Wilson, Quasi-random graphs, Combinatorica 9 (1989), 345-362.

[4] L. Carlitz and S. Uchiyama, Bounds for exponential sums, Duke Math. J. 24 (1957), $37-41$.

[5] L.E. Dickson, Linear groups: with an exposition of the Galois field theory, Teubner, Leipzig (1901), x+312pp.

[6] R.L. Graham and J.H. Spencer, A constructive solution to a tournament problem, Canad. Math. Bull. 14 (1971), 45-48.

[7] J.W.P. Hirschfeld, Projective geometries over finite fields, (2nd ed.) Clarendon, Oxford (1998), xiv+555pp.

[8] D. Kühn and D. Osthus, Hamilton decompositions of regular expanders: applications, J. Combin. Theory Ser. B 104 (2014), 1-27.

[9] R. Lidl and H. Niederreiter, Finite fields, Encyclopedia of Mathematics and its Applications 20, Cambridge University Press (1997). 
[10] W.M. Schmidt, Equations over finite fields. An elementary approach. Lecture Notes in Math. 536, Springer-Verlag (1976).

[11] S.A. Stepanov, Estimation of Kloosterman sums, (in Russian) Izv. Akad. Nauk. SSSR Ser. Mat. 35 (1971), 308-323.

[12] A.G. Thomason, Pseudo-random graphs, in "Proceedings of Random Graphs, Poznań 1985", (M. Karonski, ed.) Annals of Discrete Math. 33 (1987) 307-331.

[13] A.G. Thomason, The simplest case of Ramsey's theorem, in "Paul Erdős and his Mathematics" Bolyai Society Math. Studies 11 (2002), 667-695.

[14] A. Weil, On some exponential sums, Proc. Nat. Acad. Sci. USA 34 (1948), 204-207.

Department of Pure Mathematics and Mathematical Statistics, Centre for Mathematical Sciences, Wilberforce Road, Cambridge CB3 0WB, England

E-mail address: a.g.thomason@dpmms.cam.ac.uk 